International Mathematical Forum, 2, 2007, no. 5, 223 - 236

\title{
Spectral Theory of Ordered Pairs of the Linear Operators - acting in Different Banach Spaces and Applications ${ }^{1}$
}

\author{
M. Şahin \\ Faculty of Science and Arts, Department of Mathematics \\ University of Gaziantep, TURKEY \\ mesahin@gantep.edu.tr \\ M. B. Ragimov \\ Baku State University \\ rahimovmisir@yahoo.com
}

\begin{abstract}
Ordered pairs of linear operators $(A, B)$ from Banach space $L(X ; Y)$ of the linear bounded operators, defined on complex Banach space $X$ with the values in complex Banach space $Y$ are considered in this paper.

Notion of singular set (spectrum) of the ordered pair $(A, B)$ is introduced essentially with the help of operators bundle of the form

$$
A-\lambda B, \lambda \in \mathbb{C}
$$

and functional calculus in sections $(1,2)$. For investigation of spectral properties of the pairs $(A, B)$ we apply left and right pseudoresolvents of the pair $(A, B)$, which allow to apply theory of commutative Banach algebras and spectral theory of the operators, acting in one space. In sections $(1,2)$ we give the number of general results on the spectrum of operators pairs $(A, B)$ in dependence on some properties of the operator $B$ (connected with invertibility, finite dimensionless and etc.).
\end{abstract}

Mathematics Subject Classification: Primary 47G99, Secondary 47A99 Keywords: Left and right pseudoresolvents, quasi-similar operators

\footnotetext{
${ }^{1}$ This paper is in final form and no version of it will be submitted for publication elsewhere.
} 


\section{Spectrum and Regular Set of the Ordered Pairs of the OpERATORS}

First of all we note more applied notations. They are denoted by $\sigma(T)$ and $\rho(T)$ the spectrum and resolvent set of the linear operator $T: D(T) \subset Z \rightarrow Z$ $(D(T)$ is its domain), acting in complex Banach space $Z$.

Definition 1. Spectrum of the pair $(A, B)$, where $A, B \in L(X ; Y)$ is the collection of such complex $\lambda \in C$, for which the operator $A-\lambda B$ has not bounded inverse.

Spectrum of the pair $(A, B)$ we denote by symbol $\sigma(A, B)$. Denote by $\rho(A, B)$ the set $\mathbb{C} / \sigma(A, B)$. It is clear that the spectrum of the pair $(A, B)$ is closed, and the set $\rho(A, B)$ is open. In the case of difference of the spectrum of bounded operator (acting in one space), spectrum of the pair $(A, B)$ can be unbounded set. Moreover, it may be empty.

Example 1. We consider closed linear operator

$$
A: D(A) \subset Y \rightarrow Y
$$

acting in one Banach space $Y$ with the domain $D(A)$. In case of $X=D(A)$ and we norm the space $X$, supposing $\|x\|_{*}=\|x\|+\|A x\| \quad \forall x \in X$ (In this case $X$ becomes Banach space). Let $B x=x, x \in X, B: X \rightarrow Y$ the operator of embedding operator. Then it is clear that $\sigma(A, B)$ coincide with usual spectrum $\sigma(A)$ of the operator $A: D(A) \subset Y \rightarrow Y$. Thus, above-mentioned possibilities arise for singular set $\sigma(A, B)$ (for example, if $\sigma(A)=\emptyset$, then also $\sigma(A, B)=$ $\emptyset)$. Possible emptiness of the spectrum $\sigma(A, B)$ comes to definite difficulties and in this connection we give notion of extended spectrum of the pair $(A, B)$.

Definition 2. Extended spectrum $\tilde{\sigma}(A, B)$ of the pair $(A, B)$ is a subset from the extended complex plane $\tilde{\mathbb{C}}=\mathbb{C} \cup\{\infty\}$, which coincides with $\sigma(A, B)$, if both functions $\lambda \rightarrow B(A-\lambda B)^{-1}: \tilde{\mathbb{C}} \rightarrow L(Y), \lambda \rightarrow(A-\lambda B)^{-1} B: \tilde{\mathbb{C}} \rightarrow L(X)$ are halomorphic at the point $\infty$ and coincides with $\sigma(A, B) \cup\{\infty\}$ in opposite case. Suppose $\tilde{\rho}(A, B)=\tilde{\mathbb{C}} / \tilde{\sigma}(A, B)$.

The function $R_{A, B}(\cdot)=R(\cdot, A, B): \rho(A, B) \rightarrow L(Y ; X)$ defined by the equality.

$$
R_{A, B}(\lambda)=(A-\lambda B)^{-1}, \lambda \in \rho(A, B)
$$

is called the resolvent of operators pair $(A, B)$ from $L(X ; Y)$. From here, it is clear that

$$
R(\lambda ; A, B)-R(\mu ; A, B)=(\mu-\lambda) R(\lambda ; A, B) B R(\mu ; A, B) .
$$

From this equality particularly, we obtain for any $\lambda, \mu \in \rho(A, B)$ the following identities

$$
\begin{aligned}
R_{l}(\lambda)-R_{l}(\mu) & =(\mu-\lambda) R_{l}(\lambda) R_{l}(\mu), \\
R_{r}(\lambda)-R_{r}(\mu) & =(\mu-\lambda) R_{r}(\lambda) R_{r}(\mu),
\end{aligned}
$$


where the functions

$$
R_{l}(\lambda)=B R(\lambda ; A, B), R_{r}(\lambda)=R(\lambda ; A, B) B, \lambda \in \rho(A, B)
$$

are called the left and right pseudoresolvents for the pair $(A, B)$. Note that the functions $R_{l}: \rho(A, B) \rightarrow L(Y), R_{r}: \rho(A, B) \rightarrow L(X)$ are pseudoresolvents in sense [1] and introduced terminology is connected with it. (Note, that the function $R: U \rightarrow L(Z)$, defined on the opened set $U \subset \mathbb{C}$ is called pseudoresolvent, if $\lambda, \mu \in U$ and the following identity $R(\lambda)-R(\mu)=(\mu-\lambda) R(\lambda) R(\mu)$ takes place.

In the content of this study (without special reminding) non-emptiness of regular set of the considered ordered pairs of the operators from $L(X ; Y)$ is supposed.

Lemma 1. Left and right pseudoresolvents $R_{l}$ and $R_{r}$ of the pair $(A, B)$ are the resolvent of some operators if and only if the operator $B$ is invertible (not necessary continuous).

Proof. Let $B: X \rightarrow Y$ be invertible operator and $\operatorname{Im} B$ be its values set. Then the operator $A B^{-1}$ has the domain $D\left(A B^{-1}\right)$, equals to $\operatorname{Im} B$ and acts in one space $Y$. Let $\lambda_{0} \in \rho(A, B)$. Then

$$
R_{l}\left(\lambda_{0}\right)\left(A B^{-1}-\lambda_{0} I_{Y}\right) y=B\left(A-\lambda_{0} B\right)^{-1}\left(A-\lambda_{0} B\right) B^{-1} y=y
$$

for any $y \in \operatorname{Im} B=D\left(A B^{-1}\right)$ and

$$
\left(A B^{-1}-\lambda_{0} I_{Y}\right) R_{l}\left(\lambda_{0}\right)=\left(A-\lambda_{0} B\right) B^{-1} B\left(A-\lambda_{0} B\right)^{-1}=I_{Y} .
$$

From these equalities it follows that $\operatorname{Re}(\lambda)=\left(A B^{-1}-\lambda I_{Y}\right)^{-1}, \lambda \in \rho(A, B)$, i.e. $R_{l}$ is the resolvent of the operator $A B^{-1}$. Now let's consider the operator $C=B^{-1} A: D(C) \subset X \rightarrow X$ with domain $D(C)=\{x \in X: A x \in \operatorname{ImB}\}$. Apriority it is not clear, why $D(C) \neq\{0\}$. However the condition $\rho(A, B) \neq \emptyset$, as we'll be convinced now, guarantees that $\lambda_{0} \in \rho(A, B) \neq \emptyset$. Let $\lambda_{0} \in$ $\rho(A, B)$. Then the operator $R_{r}\left(\lambda_{0}\right)=\left(A-\lambda_{0} B\right)^{-1} B$ has the property $R_{r}\left(\lambda_{0}\right) x \in$ $D(C), \forall x \in X$ and, moreover, the set of values of this operator coincides with $D(C)$. Really, it follows from the equalities it also follows that

$$
\begin{aligned}
A R_{r}\left(\lambda_{0}\right) & =A\left(A-\lambda_{0} B\right)^{-1} B=\left(A-\lambda_{0} B+\lambda_{0} B\right)\left(A-\lambda_{0} B\right)^{-1} B \\
& =B+\lambda_{0}\left(\left(A-\lambda_{0} B\right)^{-1} B=B\left(I_{X}+\lambda_{0} R_{r}\left(\lambda_{0}\right)\right)\right. \\
\left(B^{-1} A-\lambda_{0} I_{X}\right) R_{r}\left(\lambda_{0}\right) & =I_{X}
\end{aligned}
$$

and

$$
R_{r}\left(\lambda_{0}\right)\left(B^{-1} A-\lambda_{0} I_{X}\right) x=x, \forall x \in D(C)
$$

Lemma is proved.

Directly from the Lemma 1 and its proof we obtain two following corollaries. 
Corollary 2. If the operator $B$ is invertible (not necessary continuously), then

$$
\sigma(A, B)=\sigma\left(A B^{-1}\right) \cup \sigma\left(B^{-1} A\right) .
$$

Corollary 3. If subspace

$$
D(C)=\{x \in X: A x \in \operatorname{Im} B\}
$$

consists only of zero vector and $X \neq\{0\}$ then $\sigma(A, B)=C$. Moreover, $\sigma(A, B)=C$, if $D(C)$ is finite-dimensional subspace from infinite-dimensional space $X$.

Note that if $\rho(A, B) \neq \oslash$, then for $\lambda_{0} \in \rho(A, B)$ the operator $B^{-1} A-$ $\lambda_{0} I_{X}: D(C) \subset X \rightarrow X$ will be invertible. It is not possible because of finitedimensionness of $D(C)$ and infinite-dimensionness of $X$.

The case if $B$ is non-invertible operator will be considered in the next paragraph.

\section{Operator Calculus}

Consider the pair $(A, B)$ and let $\Delta$ be open set from the extended complex plane $\tilde{\mathbb{C}}$, containing the extended spectrum $\tilde{\sigma}(A, B)$ of this pair. Denoted by symbol $H(\Delta)$ the topological algebra of halomorphic on $\Delta$ functions with topology of uniform convergent on compact subsets from $\Delta$. Always denote by $\gamma$ oriented Jordan contour, not crossing the point $\infty$ and such that $\tilde{\sigma}(A, B)$ belongs to its inside part.

For each function $f \in H(\Delta)$ we suppose

$$
T(f)=\frac{1}{2 \pi} \int_{\gamma} f(\lambda)(A-\lambda B)^{-1} d \lambda
$$

in supposition that the point $\infty$ is out of the contour $\gamma$. Formula (2.1) defines the continuous linear operator

$$
T: H(\Delta) \rightarrow L(Y, X) .
$$

Lemma 4. If $\lambda_{0} \in \rho(A, B)$, then the operator

$$
T\left(f_{0}\right)=\frac{1}{2 \pi i} \int_{\gamma} \frac{1}{\lambda-\lambda_{0}}(A-\lambda B)^{-1} d \lambda, \quad f_{0}(\lambda)=\frac{1}{\lambda-\lambda_{0}}
$$

which coincides with the operator $\left(A-\lambda_{0} B\right)^{-1}$. 
Proof. The equalities

$$
\begin{aligned}
\left(A-\lambda_{0} B\right) T\left(f_{0}\right) & =\frac{1}{2 \pi i}\left(A-\lambda_{0} B\right) \int_{\gamma} \frac{1}{\lambda-\lambda_{0}}(A-\lambda B)^{-1} d \lambda \\
& =\frac{1}{2 \pi i} \int_{\gamma} \frac{\left[A-\lambda B+\left(\lambda-\lambda_{0}\right) B\right](A-\lambda B)^{-1}}{\lambda-\lambda_{0}} d \lambda \\
& =\frac{1}{2 \pi i} \int_{\gamma} \frac{d \lambda}{\lambda-\lambda_{0}} I_{y}+\frac{1}{2 \pi i} \int_{\gamma} B(A-\lambda B)^{-1} d \lambda=I_{y}
\end{aligned}
$$

take place.

Similarly the equality $T\left(f_{0}\right)\left(A-\lambda_{0} B\right)=I_{X}$ is proved. Lemma is proved. In order to solve the difficulties, connected with the fact that $L(Y ; X)$ is not algebra for $L(Y ; X)$, we introduce two linear operators:

$$
T_{l}: H(\Delta) \rightarrow L(Y) ; T_{r}: H(\Delta) \rightarrow L(X),
$$

defined for any function $f \in H(\Delta)$ by the formulas

$$
\begin{aligned}
& T_{l}(f)=\delta f(\infty) I_{Y}+\frac{1}{2 \pi i} \int_{\gamma} f(\lambda) R_{l}(\lambda) d \lambda, \\
& T_{r}(f)=\delta f(\infty) I_{X}+\frac{1}{2 \pi i} \int_{\gamma} f(\lambda) R_{r}(\lambda) d \lambda,
\end{aligned}
$$

where $\delta=0$ or $\delta=1$ without considering the point $\infty$ whether inside $\gamma$ or outside $\gamma$. Note one more following simple connection of the operators

$$
T_{r}(f)=T(f) B ; T_{l}(f)=B T(f), f \in H(\Delta)
$$

and it is supposed that in corresponding formulas (2.2)-(2.3) contour $\gamma$ is chosen such that the point $\infty$ is out of $\gamma$ (this supposition is done, because the formula (2.1) is defined only for such contours). As $R_{l}$ and $R_{r}$ are pseudoresolvents, then using usual reasonings in the proof of properties of the operator calculus we obtain that it takes place.

Lemma 5. Operators $T_{r}: H(\Delta) \rightarrow L(X), T_{l}: H(\Delta) \rightarrow L(Y)$ are continuous homomorphisms of algebra $H(\Delta)$ and the following equalities

$$
\begin{aligned}
T_{r}(f) T(\varphi) & =T(f) B T(\varphi)=T(\varphi) B T(f)=T(\varphi) T_{l}(f) \\
T_{l}(f)(A-\mu B) & =(A-\mu B) T_{r}(f) \\
T_{l}\left(f_{0}\right) & =B R\left(\lambda_{0} ; A, B\right), T_{r}\left(f_{0}\right)=R\left(\lambda_{0} ; A, B\right) B
\end{aligned}
$$

take place for any $f, \varphi \in H(\Delta), \lambda_{0} \in \rho(A, B)$ and $f_{0}=\frac{1}{\lambda-\lambda_{0}}$. Note that the equality (2.7) follows from the equalities (2.4) and lemma 1. We'll prove the 
equality (2.6). It follows from the following equalities

$$
\begin{aligned}
\left(A-\mu_{0} B\right) T_{r}(f) & =\frac{1}{2 \pi i} \int_{\gamma}\left(A-\mu_{0} B\right) f(\lambda)(A-\lambda B)^{-1} B d \lambda \\
& =\frac{1}{2 \pi i} \int_{\gamma}\left[(A-\lambda B)+\left(\lambda-\mu_{0}\right) B\right] f(\lambda)(A-\lambda B)^{-1} B d \lambda \\
& =\frac{1}{2 \pi i} \int_{\gamma} B(A-\lambda B)^{-1} B\left(\lambda-\mu_{0}\right) f(\lambda) d \lambda \\
& =\frac{1}{2 \pi i} \int_{\gamma} \lambda B(A-\lambda B)^{-1} B f(\lambda) d \lambda-\mu_{0} T_{l}(f) B \\
& =\frac{1}{2 \pi i} \int_{\gamma} B(A-\lambda B)^{-1}(\lambda B-A+A) f(\lambda) d \lambda-\mu_{0} T_{l}(f) B \\
& =T_{l}(f)\left(A-\mu_{0} B\right)
\end{aligned}
$$

Lemma is proved.

Definition 3. Two operators $T_{1} \in L(X), T_{2} \in L(Y)$ are called similar, if there is continuously invertible operator $V \in L(X ; Y)$ such that $V T_{2}=V T_{1}$. It is clear, that similar operators $T_{1}$ and $T_{2}$ have the same spectral properties and, particularly,

$$
\sigma\left(T_{1}\right)=\sigma\left(T_{2}\right)
$$

Lemma 6. Operators $R_{r}\left(\lambda_{0}\right)$ and $R_{l}\left(\lambda_{0}\right), \forall \lambda_{0} \in \rho(A, B)$ are similar.

Proof. The equality

$$
\left(A-\lambda_{0} B\right)^{-1} R_{l}\left(\lambda_{0}\right)=R_{r}\left(\lambda_{0}\right)\left(A-\lambda_{0} B\right)^{-1}, \lambda_{0} \in \rho(A, B),
$$

takes place, such that the operators $R_{l}\left(\lambda_{0}\right)$ and $R_{r}\left(\lambda_{0}\right)$ are similar 7 .

Corollary 7. If the operator $B$ is invertible, then

$$
\sigma\left(A B^{-1}\right)=\sigma\left(B^{-1} A\right)=\sigma(A, B) .
$$

equality (2.8) follows from the equality $\sigma\left(R_{l}\left(\lambda_{0}\right)\right)=\sigma\left(R_{r}\left(\lambda_{0}\right)\right)$. Thus, this corollary comes to the corollary 2 of lemma 1 . Obtained result is applied for the investigation of spectral properties of quasi-similar operators.

Definition 4. Two operators $T_{1} \in L(X), T_{2} \in L(Y)$ are called quasi-similar, if there is (not necessary continuously) invertible operator $V: X \rightarrow Y$ such that

$$
T_{2} V=V T_{1}
$$


It is well known that two quasi-similar operators $T_{1}$ and $T_{2}$ can have noncoinciding spectrums. However from the corollary of lemma 6 we obtain that it takes place.

Theorem 8. Let $T_{1} \in L(X), T_{2} \in L(Y)$ be two quasi-similar operators, and $T_{2} B=B T_{1}$ for some invertible operator $B: X \rightarrow Y$. If the operator $T_{2} B-\lambda_{0} B$ is continuously invertible for some $\lambda \in \mathbb{C}$. Then $\sigma\left(T_{1}\right)=\sigma\left(T_{2}\right)$.

Now we'll do some limitations on the pairs of operators $(A, B)$ from $L(X, Y)$ :

1. There is the number $\lambda_{0} \in \mathbb{C}$, such that the operator $A-\lambda_{0} B$ is continuously invertible, i.e. $\rho$ regular set $\rho(A, B) \subset \mathbb{C}^{\prime}$ is non-empty

2. Operators $R_{r}(\lambda ; A, B), \lambda \in \rho(A, B)$ from $L(X)$ and $R_{l}(\mu ; A, B)$ from $L(Y), \mu \in \rho(A, B)$ are mutually commutative.

The least closed sub-algebras of the operators from $L(X)$ and $L(Y)$, containing all operators $R_{r}(\lambda ; A, B), \lambda \in \rho(A, B), R_{l}(\mu ; A, B), \mu \in \rho(A, B)$ and identical operators $I_{X}$ and $I_{Y}$ correspondingly are denoted by $B_{r}$ and $B_{l}$.

It is clear that $B_{r}$ and $B_{l}$ are commutative Banach algebras. Now we'll formulate one applied by us result on the spectral properties of pseudoresolvent ([2], theorem 5.8.4).

Theorem 9. Let $R: U \rightarrow L(X)$ is pseudoresolvent, defined on opened set $U$ from $C$. Let $B$ is the least complete (i.l. containing continuous inverse to each continuously invertible in $L(X)$ operator from $B$ ) subalgebra from $L(X)$, containing the operator $I_{X}$ and all operators $R(\lambda), \lambda \subset U$, and $\mathcal{M}$ is its space of maximal ideals.

Then there is continuous function $\alpha: \mathcal{M} \rightarrow \tilde{\mathbb{C}}$ such that for all $\lambda \subset U$ it takes place the equality:

$$
R(\lambda)(M)=(\lambda-\alpha(M))^{-1}, M \in \mathcal{M}
$$

Further, $\sigma=\{\alpha(M): M \in \mathcal{M}\}$ is closed subset of $\tilde{\mathbb{C}}$ and $\sigma \cap U=\emptyset$.

From Theorem 9 it follows that the function $\alpha: \mathcal{M} \rightarrow \overline{\mathbb{C}}$ constructs homeomorphism between $\mathcal{M}$ and closed subset $\sigma$ from $\tilde{\mathbb{C}}$, and it allows to identify the space $\mathcal{M}$ with subset $\sigma$ from $\tilde{\mathbb{C}}$.

We apply theorem 9 and now mention notation about pseudoresolvents $R_{l}$ and $R_{r}$ (without any limitations on the operator $B$ ) as it was done above, denoting by symbols $B_{l}$ and $B_{r}$ the least subalgebras from $L(Y)$ and $L(X)$ containing $I_{Y}, R_{l}(\lambda)$ and $I_{X}, R_{r}(\lambda), \lambda \in \rho(A, B)$ correspondingly. The functions which are defined according to formula (2.9) are denoted by $\alpha_{l}: \mathcal{M}_{l} \rightarrow \tilde{\mathbb{C}}$ and $\alpha_{r}: \mathcal{M}_{r} \rightarrow \overline{\mathbb{C}}$ the functions, ( $\mathcal{M}_{l}$ and $\mathcal{M}_{r}$ are the spaces of maximal ideals of algebras $B_{l}$ and $B_{r}$ correspondingly). If $\lambda_{0} \in \rho(A, B)$, then $\sigma\left(R_{l}\left(\lambda_{0}\right)\right)=\sigma\left(R_{r}\left(\lambda_{0}\right)\right)$ according to lemma 6 and because of it the sets $\alpha_{l}\left(\mathcal{M}_{l}\right)$ and $\alpha_{r}\left(\mathcal{M}_{r}\right)$ coincide, i.e.. the space of maximal ideals of algebras $B_{l}$ and $B_{r}$ are homeomorphic (coincide). So, from the obtained reasonings it follows [2]. 
Lemma 10. Spaces of maximal ideals $\mathcal{M}_{l}$ and $\mathcal{M}_{r}$ of Banach algebras $B_{l}$ and $B_{r}$ are homeomorphic to closed subset $\sigma$ from the extended complex plane $\tilde{\mathbb{C}}$, which has the property $\sigma \cap \tilde{\rho}(A, B)=\emptyset$.

Definition 5. Let the Banach spaces $X$ and $Y$ allow the decompositions $X=$ $X_{1} \oplus X_{2}, Y=Y_{1} \oplus Y_{2}$ in direct sum of its closed subspaces $X_{i}, Y_{i}, i=1,2$. We say that the pair $(A, B)$ allows reducing relatively the painted decomposition of spaces, if $A X_{i} \subset Y_{i}, \quad B X_{i} \subset Y_{i}, i=1,2$. In this case we'll write

$$
(A, B)=\left(A_{1} \oplus A_{2}, B_{1} \oplus B_{2}\right)=\left(A_{1}, B_{1}\right) \oplus\left(A_{2}, B_{2}\right),
$$

where $A_{i}: X_{i} \rightarrow Y_{i}, i=1,2$ is narrowing of $A$ on $X_{i}$ and $B_{i}: X_{i} \rightarrow Y_{i}, i=$ 1,2 is narrowing of $B$ on $X_{i}$. Decomposition (2.10) is called the decomposition of $\operatorname{pair}(A, B)$.

Obtained by this way pairs $\left(A_{i}, B_{i}\right), i=1,2$ are called the reduced pairs relatively the pointed decomposition of spaces $X, Y$.

Notation 1. Let $P_{i} \in L(X), Q_{i} \in L(Y), i=1,2$ be projectors, providing decompositions $X=X_{1} \oplus X_{2}, Y=Y_{1} \oplus Y_{2}$, i.l. $P_{i} X=X_{i}, i=1,2, Q_{i} Y=$ $Y_{i}, i=1,2$. Then it is called, that the pair $(A, B)$ is reducing relatively the pointed decomposition of spaces if and only if the equalities take place:

$$
A P_{i}=Q_{i} A, B P_{i}=Q_{i} B, i=1,2
$$

Notation 2. If $(A, B)=\left(A_{1}, B_{1}\right) \oplus\left(A_{2}, B_{2}\right)$, then it takes place the equalities

$$
\begin{aligned}
R(\lambda: A, B) & =(A-\lambda B)^{-1}=\left(A_{1}-\lambda B_{1}\right)^{-1} \oplus\left(A_{2}-\lambda B_{2}\right)^{-1}, \\
R_{l}(\lambda) & =B(A-\lambda B)^{-1}=B_{1}\left(A_{1}-\lambda B_{1}\right)^{-1} \oplus B_{2}\left(A_{2}-\lambda B_{2}\right)^{-1}, \\
R_{r}(\lambda) & =(A-\lambda B)^{-1} B=\left(A_{1}-\lambda B_{1}\right)^{-1} B_{1} \oplus\left(A_{2}-\lambda B_{2}\right)^{-1} B_{2} .
\end{aligned}
$$

Now we'll prove the following theorem on absentness of the point $\infty$ in spectrum $\tilde{\sigma}(A, B)$ of operators pair $(A, B) \in L(X, Y)$.

Theorem 11. Spectrum $\tilde{\sigma}(A, B)$ of the pair $(A, B)$ of operators $A, B: X \rightarrow$ $Y$ doesn't contain the point $\infty$ (particularly, it is bounded) if and only if the pair $(A, B)$ allows the reducing of the form (2.10) relatively some decomposition

$$
X=X_{1} \oplus X_{2} ; Y=Y_{1} \oplus Y_{2}
$$

and the following conditions are fulfilled: 1) operator $B_{1}: X_{1} \rightarrow Y_{1}$ is continuously invertible and 2) $A_{2}: X_{2} \rightarrow Y_{2}$ is continuously invertible and $\left(A_{2}^{-1} B_{2}\right)^{2}=$ 0 .

Proof. Let $\infty \bar{\epsilon} \sigma(A, B)$. Then the set of $\sigma(A, B)$ is compact. Consider the operator

$$
C=\frac{1}{2 \pi i} \int_{\gamma}(A-\lambda B)^{-1} d \lambda
$$


where $\gamma$ is Jordan contour of $\sigma(A, B)$. We'll show that the operators $C B=$ $P_{1} \in L(X)$ and $B C=Q \in L(Y)$ are projectors, satisfying the conditions

$$
A P_{1}=Q_{1} A, B P_{2}=Q_{2} B
$$

Consider the operator $P_{1}=C B$. Then the operator

$$
\begin{aligned}
P_{1} & =C B=\frac{1}{2 \pi i} \int_{\gamma}(A-\lambda B)^{-1} B d \lambda \\
& =\frac{1}{2 \pi i} \int_{\gamma} R_{r}(\lambda) d \lambda=T_{r}(1)
\end{aligned}
$$

is projector on the property of functional calculus for pseudoresolvent (functions $\lambda \rightarrow R_{l}(\lambda), \lambda \rightarrow R_{r}(\lambda)$ are halomorphic at the point $\left.\infty\right)$. Similarly it is proved that the operator

$$
Q_{1}=B C=T_{l}(1)
$$

is projector.

We have to use the equality (2.6) from lemma 5, from which it follows the equality (2.12) and, consequently, the equality (2.11) (if we take into account that $\left.P_{1}+P_{2}=I_{X}, Q_{1}+Q_{2}=I_{Y}\right)$. Thus from the notation 1 it follows that the pair $(A, B)$ allows the reducing of the form (2.10) relatively the decomposition of the form (2.12), where

$$
X_{i}=P_{i} X, Y_{i}=Q_{i} Y, i=1,2 .
$$

From the pointed equalities $P_{1}=C B, Q_{1}=B C$ it follows that the operator $B_{1}: X \rightarrow Y_{1}$ is continuously inverse, and $A_{2}^{-1} B_{2}$ is zero operator. Really, on Liouville theorem the operator $\left(A_{2}-\lambda B_{2}\right)^{-1} B_{2}$ is constant operator $C_{2} \in$ $L(X)$. We obtain from here the following equalities

$$
\begin{aligned}
\left(A_{2}-\lambda B_{2}\right)^{-1} B_{2} & =C_{2}=\left(I_{x_{2}}-\lambda A_{2}^{-1} B_{2}\right)^{-1} A_{2}^{-1} B_{2} \\
& =\left(I_{x_{2}}-\lambda A_{0}\right)^{-1} A_{o}
\end{aligned}
$$

where $A_{0}=A_{2}^{-1} B_{2}$. Because of this $C_{2}=C_{2}-\lambda C_{2} A_{0}$ and $A_{0}=C_{2}$. Consequently, $C_{2} A_{0}=A_{0}^{2}=0=\left(A_{2}^{-1} B_{2}\right)^{2}=0$. Now let the pair $(A, B)$ allow the reducing relatively some decomposition of the form (2.12), operators $B_{1}: X_{1} \rightarrow Y_{1}, A_{2}: X_{2} \rightarrow Y_{2}$ are continuously invertible and $\left(A_{2}^{-1} B_{2}\right)^{2}=0$. Then it is clear that

$$
R_{r}(\lambda)=(A-\lambda B)^{-1} B=\left(A_{1}-\lambda B_{1}\right) B_{1} \oplus\left(A_{2}-\lambda B_{2}\right)^{-1} B_{2} .
$$

As $\left(A_{1}-\lambda B_{1}\right)^{-1} B_{1}=\left(A_{1} B_{1}^{-1}-\lambda_{Y_{1}}\right)^{-1}$ is resolvent of bounded operator $A_{1} B_{1}^{-1}$ then this function is halomorphic in $\infty$. Further

$$
\begin{aligned}
\left(A_{2}-\lambda B_{2}\right)^{-1} B_{2} & =\left(I_{X_{2}}-\lambda A_{2}^{-1} B_{2}\right)^{-1} A_{2}^{-1} B_{2} \\
& =\left(I_{X_{2}}+\lambda A_{2}^{-1} B_{2}\right) A_{2}^{-1} B_{2}=A_{2}^{-1} B_{2} .
\end{aligned}
$$


Because of it the function $R_{r}(\lambda)$ is halomorphic at the point $\infty$, i.e. the point $\infty$ doesn't belong to $\tilde{\sigma}(A, B)$.

Theorem is proved.

Corollary 12. $\sigma(A, B)=\sigma\left(A_{1}, B_{1}\right)$, if $\infty \bar{\epsilon} \tilde{\sigma}(A, B)$.

Theorem 13. Mentioned in lemma 10 set $\sigma$ coincides with extended spectrum $\tilde{\sigma}(A, B)$ of pair $(A, B)$, and for any $\lambda_{0} \in \rho(A, B)$ the equalities

$$
\sigma\left(R_{l}\left(\lambda_{0}\right)\right)=\sigma\left(R_{r}\left(\lambda_{0}\right)\right)=\left\{\frac{1}{\lambda-\lambda_{0}}: \lambda \in \tilde{\sigma}(A, B)\right\}
$$

take place.

Proof. If $\infty \bar{\in} \tilde{\sigma}(A, B)$, then from previous theorem we obtain that (see also its corollary) without limitation of generality we can consider the operator $B$ continuously invertible (otherwise we have to consider the reducing pair with invertible operator $B_{1}$ ). However in the case of invertible $B$, theorem 13 is obvious. Now we consider the case, when $\infty \bar{\epsilon} \tilde{\sigma}(A, B)$. Let $\lambda_{0}$ be some point from $\rho(A, B) \neq \emptyset$. From the equality

$$
(A-\lambda B)=\left(A-\lambda_{0} B\right)\left(I_{X}-\left(\lambda-\lambda_{0}\right) R_{r}\left(\lambda_{0}\right)\right)
$$

it follows that $\lambda_{0} \neq \lambda \in \rho(A, B)$ if and only if the number $\left(\lambda-\lambda_{0}\right)^{-1}$ doesn't belong to spectrum of operator $R_{r}\left(\lambda_{0}\right)$.

From the formula (1.1) and lemma 10 we obtain that $\lambda \tilde{\epsilon} \sigma$ if and only if $\lambda \in \rho(A, B)$. On the condition $\infty \in \tilde{\sigma}(A, B)$, and according to theorem 13 the operator $R_{r}\left(\lambda_{0}\right)$ is not invertible, i.e. $\infty \in \sigma$. Similar reasonings are done for $R_{l}\left(\lambda_{0}\right)$. Theorem is proved.

Corollary 14. If $B$ is finite-dimensional operator, then $\sigma(A, B)$ is finite set, the number of elements of which is not more that the rang of operator $B$ (dimension of the set of values of operator $B$ ). If $X$ is infinite-dimensional space and co-dimension of the values set of the operator $A$ is equal to $\infty$, then $\infty \bar{\epsilon} \sigma(A, B)$.

Proof. Let $\lambda_{0} \in \rho(A, B)$. Then the set $\tilde{\sigma}(A, B)$ homeomorphic $\sigma\left(R_{l}\left(\lambda_{0}\right)\right)$. As the operator $R_{l}\left(\lambda_{0}\right)=B\left(A-\lambda_{0} B\right)^{-1}$ is finite dimensional, the number of the points in $\sigma(A, B)$ is not more than the rang of operator $R_{l}\left(\lambda_{0}\right)$ and, consequently, the rang of operator $B$.

The second part of corollary is direct corollary of theorem 13. Corollary is proved. From the reasonings, similar processes are done for the proof of corollary 7 , we obtain once more.

Corollary 15. If $B$ is absolutely continuous operator, then $\tilde{\sigma}(A, B)$ is not more than countable set with unique possible limit point, which equals to $\infty$.

Theorem 16. For any function $f$ from $H(\Delta)$ the equalities

$$
\sigma\left(T_{r}(f)\right)=\sigma\left(T_{l}(f)\right)=f(\tilde{\sigma}(A, B))=\{f(\lambda): \lambda \in \tilde{\sigma}(A, B)\}
$$

take place. 
Proof. Consider the Banach algebra $B_{r}(\subset L(X))$, introduced by us earlier. Then from the formula (2.8) for any maximal ideal $m \in \mathcal{M}_{r}=\tilde{\sigma}(A, B)=\sigma$ it takes place the equality

$$
\begin{aligned}
T_{r}(f)(M) & =\delta f(\infty)+\frac{1}{2 \pi i} \int_{\gamma} f(\lambda) \cdot \frac{1}{\left(\lambda-\alpha_{r}(M)\right)} d \lambda \\
& =f\left(\alpha_{r}(M)\right) .
\end{aligned}
$$

As the operator $T_{r}(f)$ belongs to algebra $B_{r}$,

$$
\sigma\left(T_{r}(f)\right)=\left\{T_{r}(f)(M): M \in \mathcal{M}_{r}=\sigma(A, B)\right\}=f(\tilde{\sigma}(A, B)) .
$$

The equality $\sigma\left(T_{l}(f)\right)=f(\tilde{\sigma}(A, B))$ is proved similarly. Theorem is proved.

\section{Application}

In this part we consider the linear differential equation of the second order of the following type:

$$
A \ddot{X}+B \dot{X}+C X=\psi(t)
$$

where $A: D(A) \subset X \rightarrow X, B: D(B) \subset X \rightarrow X, C: D(C) \subset X \rightarrow X$ are linear options, acting in complex Banach space $X$ and having the definition domains $D(A), D(B), D(C)$ resp. such that the supspace $X_{0}=D(A) \cap$ $D(B) \cap D(C)$ is dense in $X$. We make various assumptions about the function $\psi:(a, f) \rightarrow X$. Nevertheless one can obtain the most important results in that case, where $\psi$ belongs to the Banach space $C(R ; X)$ of the $X$ valued continuous on $R \mathrm{R}$ functions and especially to the subspace of continuous almost periodical functions [3]

The case $A, B, C \in L(X ; Y)$ and $\psi \in B(R ; Y)$ is considered separately. The equation (3.2) for $\psi \in C(R ; X)$ is conveniently to consider as the operator equation of the following type

$$
L_{X}=\psi,
$$

where $L: D(L) \subset C(R ; X) \rightarrow C(R ; X)$ is linear operator

$$
L_{X} \equiv A \ddot{X}+B \dot{X}+C X
$$

with appropriate definition domain $D(L)$.

The problem to determine the definition domain $D(L)$ is very complex in the case when at least on of $A, B, C$ is non-bounded operator.

To determine $D(L)$ one ought to make some additional assumption on the operators $A, B, C$ considering the operator-valued function $H$ defined on $R$, with values in the set of linear closed operators. This function is determined by the formula $H(\lambda)=\lambda^{2} A+i B \lambda+C$ under the assumption that each of operators $H(\lambda): D(H(\lambda)) \subset X \rightarrow X$ is closed extension with $X_{0}=$ $D(A) \cap D(B) \cap D(C)$ and $D(H(\lambda)) \supset D(A), D(H(\lambda)) \supset D(B)$. Let us make the main assumptions respected to the bundle of operators $H(\lambda), \lambda \in$ 
$C$ : There exists such complex number $Z_{0}$, that the operator-valued function $H_{0}(\lambda)=H(\lambda)+Z_{0} I$ satisfies the conditions:

Each of operators $H_{0}(\lambda), \lambda \in R$ has continuous inverse one and $H_{0}^{-1}(\lambda)$ admits the estimation of the following type:

$$
\left\|H_{0}^{-1}(\lambda)\right\| \leq \frac{\text { const }}{(1+|\lambda|)^{1+\alpha}}, \forall \lambda \in R
$$

where $\alpha>0$ is some number and const is absolute constant, independent on $\lambda \in R$;

Operators $A H_{0}^{-1}(\lambda), B H_{0}^{-1}(\lambda)$ are bounded and

$$
\left\|A H_{0}^{-1}(\lambda)\right\| \leq \frac{\text { const }}{(1+|\lambda|)},\left\|B H_{0}^{-1}(\lambda)\right\| \leq \text { const }
$$

for each $\lambda \in R$ (const-the absolute constant, independent on $\lambda \in R$ ) the trying of the conditions (3.2)-(3.3) is simple than the trying the correctness condition of the Cauchy problem for respective homogeneous equation $(3.2) .(\psi=0)$.

Note : In the case $A, B, C \in L(X ; Y)$ the respective function $H: R \rightarrow$ $L(X ; Y)$ is defined

$$
H(\lambda)=(i \lambda)^{2} A+(i \lambda) B+C(*)
$$

and about this function one ought to make the following assumption, different from above ones:

Suppose that the space $X$ is continuously imbedded into $Y$ and there exists $Z_{0} \in C$ such that function $H_{0}(\lambda)=H(\lambda)+Z_{0} I_{*}$, where $I_{*}: X \rightarrow Y$ the imbedding operator has continuous inverse for each $\lambda \in R$ and analogs of the conditions (3.2)-(3.3) holds

$$
\begin{aligned}
& \left\|H_{0}^{-1}(\lambda)\right\|=\frac{\text { const }}{(1+|\lambda|)^{1+\alpha}}, \quad \alpha>0(3.2) \prime \\
& \left\|A H_{0}^{-1}(\lambda)\right\|=\frac{\text { const }}{1+|\lambda|}, \quad\left\|B H_{0}^{-1}(\lambda)\right\| \leq \text { const, }(3.3) \prime
\end{aligned}
$$

where $\lambda \in R$ and const is absolute constant, $H_{0}^{-1}: R \rightarrow L(Y, X)$, $A H_{0}^{-1}(\lambda), B H_{0}^{-1}(\lambda) \in L(Y), \quad \lambda \in R$.

Lemma 17. The function $H_{0}(\lambda)$ satisfying the continuous (3.2)-(3.3) (resp. $\left.H_{0}: R \rightarrow L(X ; Y)\right)$ satisfying conditions (3.2)-(3.3) has the property: the exists the continuous operator-valued summable function $G: R \rightarrow L(X)$ (resp. $G: R \rightarrow L(Y ; X))$ such that

$$
H_{0}^{-1}(\lambda)=\int_{-\infty}^{\infty} G(t) e^{-i \lambda t} d t, \lambda \in R
$$

i.e. the function $H_{0}^{-1}(\lambda)$ is the Fourier transformation of the summable function $G(t)\left(\int_{-\infty}^{\infty}\|G(t)\| d t=+\infty\right)$.

Proof. Set

$$
G(t)=\int_{-\infty}^{\infty} H_{0}^{-1}(\lambda) e^{i \lambda t} d \lambda, t \in R
$$


From the condition of summability of the function $H_{0}^{-1}(\lambda)$ (which follows from the condition (3.2) or (3.2) $)$ ) follows that this function is continuous and bounded. (Moreover, $\|G(t)\| \rightarrow 0$ as $|t| \rightarrow \infty$ ). From the definition of the function $H_{0}^{-1}(\lambda)$ and conditions (3.3) or (3.3) $/$ immediately follows that

$$
\frac{d H_{0}^{-1}(\lambda)}{d \lambda}=H_{0}^{-1}(\lambda)(-2 A \lambda+i B) H_{0}^{-1}(\lambda)
$$

and

$$
\left\|\frac{d H_{0}^{-1}(\lambda)}{d \lambda}\right\| \leq \text { const }\left\|H_{0}^{-1}(\lambda)\right\|\left(\frac{|\lambda|}{1+|\lambda|}+1\right) \leq \frac{\text { const }}{1+|\lambda|}, \lambda \in R,
$$

i.e. the function $H_{0}^{-1}(\lambda)$ is continuously differentiable amd summable on $R$.One can analogously prove that it has the second derivative $\frac{d^{2} H_{0}^{-1}}{d \lambda^{2}}$ and

$$
\left\|\frac{d^{2} H_{0}^{-1}(\lambda)}{d \lambda^{2}}\right\| \leq \frac{\text { const }}{(1+|\lambda|)^{\alpha}} .
$$

Taking it into account from (3.4) one can obtain the inequality

$$
-t^{2} G(t)=\int_{-\infty}^{\infty} G(t) x_{0} e^{i \lambda_{o} t} d t
$$

belongs to $D(L)$ and therefore from this equality follows that the function $y_{0} e^{i \lambda t}$ belongs to $D(L)$ if $y_{0} \in D\left(H_{0}\left(\lambda_{0}\right)\right)$. For $D\left(H_{0}\left(\lambda_{0}\right)\right)=D\left(H\left(\lambda_{0}\right)\right)$, the function $y_{0} e^{i \lambda_{0} t}$ belongs to $D(L)$ if $y_{0} \in D\left(H\left(\lambda_{0}\right)\right)$.

Definition 6. Continuous bounded function $\varphi: R \rightarrow X$ is called the generalized bounded solution of the equation (3.2) if it satisfies the equation

$$
\varphi(t)=\int_{-\infty}^{\infty} G(t-s)\left(\psi(s)+z_{0} \varphi(s)\right) d s
$$

This approach to the definition of generalized solution of the equation (3.2) is very convinied from operator viewpoints. The main reason of it is that the conditions (3.2)-(3.3) on bundle make possible to define the natural definition domain $D(L)$ of the operator $L: D(L) \subset C(R ; X) \rightarrow C(R ; X)$ in the Banach space $C(R ; X)$ of the bounded on $R$ functions with values on $X$, defined by the differential expression:

$$
L X \equiv A \ddot{X}+B \dot{X}+C X .
$$

In order to define $D(L)$ we produced as follows: firstly we define the definition domain of the operator $L+Z_{0} I$ and then write : $D(L)=D\left(L+Z_{0} I\right)$.

Definition 7. The Beurling spectrum of a continuous bounded function $\varphi$ : $R \rightarrow X$ is defined to be the set of common zeros of the Fourier transforms of the functions in the set

$$
\left\{g \in L_{1}(R): g * \varphi=0\right\}
$$


where $L_{1}(R)$ is the Banach space of integrable complex-valued functions on $R$ with convolution of functions as multiplication. The Beurling spectrum of $\varphi$ is denoted by $S(\varphi)$.

It is not hard to show that the Beurling spectrum of $\varphi$ coincides with the support of its Fourier transform (if it is regarded as a generalization function of slow grouth).

Definition 8. The set of non-almost-periodic of a continuous bounded function $\varphi: R \rightarrow X$ is defined to be the common set of zeros of the Fourier transforms of the functions in the set

$$
\left\{g \in L_{1}(R): g * \varphi \text { is an } H \text { P function }\right\}
$$

The set of non-almost periodicity of a function $\varphi$ is denoted by $S_{0}(\varphi)$.

Definition 9. The singular set of the pencil $M(\lambda), \lambda \in R$, defined by $\left(^{*}\right)$ is defined to be the complement in $R$ of the set $\{\lambda \in R\}$ : the operator $M(\lambda)$ has a continuous inverse.

The singular set is closed and is denoted by $S(M)$.

Theorem 18. The set $S_{0}(\varphi)$ of non-almost-periodicity of a generalized solution $\varphi: R \rightarrow X$ of equation (3.2) is contained in the singular set of $S(M)$ of the pencil $M(\lambda), \lambda \in R$. In particular, $\varphi$ is an $H P$ function if $S(M)=\emptyset$. Moreover, $S_{0}(\varphi) \subset S(M) \cup S(\varphi)$.

\section{REFERENCES}

[1] Yosida K. Functional Analysis. Springer-Verlag Berlin, 1965.

[2] Hille E, Follips R.M. Functional Analysis and semi-groups. Moscow, IL 1962.

[3] Baskakov A.G. Spectral Criterions for Being Almost Periodical Solutions of Functional Equations. Math. Zametki Vol:24, No:2. (1978), 195-206.

Received: January 19, 2006 